\title{
Can neutrophil-to-lymphocyte, platelet-to-lymphocyte and monocyte-to-lymphocyte ratios be useful to the diagnosis of aseptic loosening after total knee arthroplasty?
}

\author{
Bozgeyik $\mathrm{B}^{1}$, Tekin $\mathrm{SB}^{2}$, Mert $\mathrm{A}^{3}$ \\ Kadirli State Hospital Department of Orthopedic Surgery Osmaniye, Turkey. \\ bahribozgeyik@gmail.com
}

\begin{abstract}
BACKGROUND: The aim of this study is to determine the role of hematological parameters - neutrophil/ lymphocyte, platelet/lymphocyte, and monocyte/lymphocyte ratios - in the diagnosis of aseptic loosening after total knee arthroplasty.

METHODS: This study retrospectively analyzed the data of 244 patients who had primary total knee arthroplasty and 66 patients with aseptic loosening developed after total knee arthroplasty. The white blood cell counts, neutrophil/lymphocyte ratio, platelet/lymphocyte ratio, monocyte/lymphocyte ratio and c-reactive protein levels in both groups were determined using the results of venous blood samples collected during preoperative preparation and compared between the groups.

RESULTS: Our study findings reveal that the monocyte/lymphocyte ratio of the group with aseptic loosening was statistically significantly different from that of the patient group who had primary total knee arthroplasty $(p=0.02)$. Furthermore, although c-reactive protein levels are not high enough to suggest systemic inflammation, the difference between the groups is statistically significant $(p=0.01)$.

CONCLUSIONS: No hematological parameter that could be used in the diagnosis of aseptic loosening has been defined in the literature so far. This study demonstrated that the monocyte/lymphocyte ratio could be a helpful parameter in the diagnosis of aseptic loosening (Tab. 1, Fig. 1, Ref. 28). Text in PDF www.elis.sk KEY WORDS: knee, arthroplasty, revision, monocyte, neutrophil.
\end{abstract}

\section{Introduction}

Total knee arthroplasty is an effective surgical option for the treatment of osteoarthritis (1). After this satisfactory surgical procedure some complications may occur. An example is the loosening that develops following total knee arthroplasty. Aseptic loosening is one of the most common conditions requiring revision after total knee arthroplasty $(2,3)$. Loosening that occurs in the early period after prosthesis implantation is usually associated with inadequate fixation, while the loosening that occurs in the following years is associated with bone resorption (4). This clinical condition can sometimes be difficult to diagnose until the migration of prosthetic components is observed $(5,6)$. Radiography is insufficient for the diagnosis of loosening in the early stages, and it is more sensitive to the malposition of the prosthetic components and in detection of fractures $(7,8)$. Bone scintigraphy with technetium helps the diagnosis but it is not specific to aseptic loosening $(6,9)$.

${ }^{1}$ Kadirli State Hospital Department of Orthopedic Surgery Osmaniye, Turkey, ${ }^{2} 25$ Aralik State Hospital Department of Orthopedic Surgery Gaziantep, Turkey, and ${ }^{3}$ Ömer Halis Demir University Hospital Department of Orthopedic Surgery Nigde, Turkey

Address for correspondence: B. Bozgeyik, küçük kizilhisar district gedikardi area 72/B-40, Poste code: 27470 Şahinbey, Gaziantep, Turkey. Phone: 05079711156
Given that the diagnosis is mostly based on clinical observation, the difficulty in establishing the diagnosis indicates a necessity of auxiliary diagnostic tools. Therefore, the present study investigated the diagnostic assistance of biochemical parameters. In addition, the selection of eligible patients is critical to establishing the diagnosis of aseptic loosening and the requirement of revision arthroplasty (10).

To the best of our knowledge, the effect of hematological parameters on the diagnosis of aseptic loosening has not been described in the literature so far. Our aim is to examine the diagnostic value of hematological parameters, namely neutrophil/ lymphocyte (NLR), platelet/lymphocyte (PLR), and monocyte/ lymphocyte (MLR) ratios, in aseptic loosening.

\section{Materials and methods}

This study retrospectively analyzed the data of patients who presented with the diagnosis of knee osteoarthritis and had undergone primary total knee arthroplasty between January 2005 and January 2020, as well as the data of patients who had undergone revision after total knee arthroplasty due to aseptic loosening diagnosed at follow-up after primary total knee arthroplasty. Our study was approved by the ethics committee and planned in accordance with the Declaration of Helsinki. 
Tab. 1. Demographic data of group 1 and group 2 and comparison between groups.

\begin{tabular}{|c|c|c|c|c|}
\hline & & Group 1 & Group 2 & $\mathrm{p}$ \\
\hline & No & 66 & 244 & \\
\hline \multirow{3}{*}{ Gender } & Male & $18(27 \%)$ & $44(18 \%)$ & 0.24 \\
\hline & Female & $48(73 \%)$ & $200(82 \%)$ & \\
\hline & Age & $65.51(49-79)$ & $66.9(48-90)$ & 0.37 \\
\hline \multirow{2}{*}{ Side } & Right & $40(60 \%)$ & $164(67 \%)$ & 0.70 \\
\hline & Left & $26(40 \%)$ & $80(33 \%)$ & \\
\hline \multirow{5}{*}{ Comorbidity } & None & $48(73 \%)$ & $168(68 \%)$ & 0.418 \\
\hline & 1 & $18(27 \%)$ & $64(27 \%)$ & \\
\hline & $\geq 2$ & 0 & $12(5 \%)$ & \\
\hline & CRP & $0.78(0.05-3.9)$ & $0.49(0.03-3.6)$ & 0.01 \\
\hline & White blood cells & $7927(3800-11400)$ & $7863(3800-14100)$ & 0.868 \\
\hline \multirow{3}{*}{$\begin{array}{l}\text { Hematological } \\
\text { parameters }\end{array}$} & NLR & $2.14(0.85-3.9)$ & $1.95(0.43-5.27)$ & 0.26 \\
\hline & PLR & $131.57(67.37-271)$ & $120.77(39.48-319)$ & 0.25 \\
\hline & MLR & $0.28(0.12-0.5)$ & $0.22(0.07-0.5)$ & 0.02 \\
\hline
\end{tabular}

and percentage for categorical variables. For statistical analysis between groups oneway ANOVA test followed by the post-hoc Dunn's test. A further ROC analysis was conducted for MLR to analyze the area under the curve as well as sensitivity and specificity. Analyses were conducted using SPSS Version 22.0. The level of $\mathrm{p}<0.05$ was considered significant.

\section{Results}

Out of 310 study patients, 66 patients who had undergone revision after total knee arthroplasty with the diagnosis of aseptic loosening were evaluated as part of group 1 , and 244 patients who had undergone pri-

The study sample included 310 patients comprising 244 patients who had undergone primary total knee arthroplasty with the diagnosis of osteoarthritis and had accessible follow-up data, and 66 patients who had undergone revision after total knee arthroplasty due to the results of bone scintigraphy with technetium that were compatible with aseptic loosening while having no growth in their intraoperative microbiological culture samples. Aseptic loosening was diagnosed with bone-scan scintigraphy with technetium and x-rays. Excluded were patients scheduled for revision after total knee arthroplasty with the diagnosis of periprosthetic infection incompatible with aseptic relaxation, patients with c-reactive protein (CRP) levels suggestive of infection, patients diagnosed with autoimmune disease, patients under follow-up with the diagnosis of malignancy or those receiving immunosuppressive agents, and patients whose postoperative culture samples were positive for bacterial growth. All patients were operated by one surgeon.

The study patients were evaluated as part of one of two groups. The analyzed variables included age, gender, operated side, presence and number of comorbidities, white blood cell count, CRP levels, and neutrophil, lymphocyte, monocyte, and platelet counts from venous blood samples collected during preoperative preparation, as well as NLR, PLR, and MLR. Furthermore, the time from primary total knee arthroplasty to aseptic loosening was ascertained.

\section{Patients and care}

All patients were operated under spinal anesthesia. A tourniquet was used in all operations. Cefazolin sodium was administered for prophylaxis 30-60 minutes before applying the tourniquet and its administration continued intravenously for one postoperative day. In addition, patients were mobilized with full weight-bearing on postoperative day 1. Low molecular-weight heparin (0.4 IU) was being administered subcutaneously for prophylaxis for one month.

\section{Statistical analysis}

Descriptive statistics of the study data were presented as mean and standard deviation for numerical variables and frequency mary total knee arthroplasty were part of group 2 . There were 248 (80\%) women and $62(20 \%)$ men among 310 patients, and their mean age was $66.61(48-90)$ years. The mean time from primary total knee arthroplasty to aseptic loosening was $76.72(12-180)$ months. The comparison of data between the groups revealed no statistically significant differences in gender, operated side, age, comorbidity, and white blood cell count. When CRP levels were compared between the groups, the group with aseptic loosening was found to have a statistically significant change $(p=0.01)$. In addition, there was a statistically significant inter-group difference in MLR, one of the hematological parameters $(p=0.02)$. There was no statistically significant inter-group difference in NLR and PLR ( $p>0.05)$ (Tab. 1).

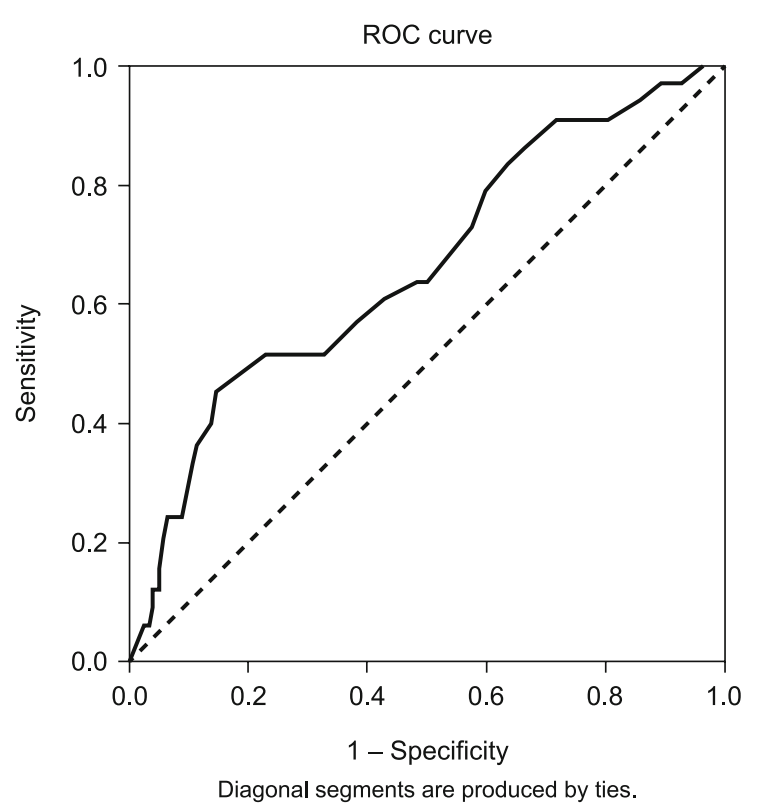

Fig. 1. ROC analysis curves for MLR. AUC = 0.66; sensitivity $77 \%$, specificity $74 \%$. ROC - receiver operating characteristic, AUC - area under curve, MLR - monosit/lymphocyte ratio. 
880-883

The ROC analysis for MLR identified a cut-off value of 0.23 (sensitivity $77 \%$ and specificity $74 \%$ ) (Fig. 1).

\section{Discussion}

The present study examined the diagnostic value of hematological parameters in patients with aseptic loosening, which is one of the potential complications following total knee arthroplasty. Our findings showed a significant relationship between MLR, one of the parameters, and aseptic loosening $(p=0.02)$. To the best of our knowledge, this is the first study to analyze the hematological parameters used in the diagnosis of aseptic loosening.

Although the pathophysiology of aseptic loosening has not been fully clarified, research indicates the presence of macrophages and giant cells in the femoral cortex in the samples from the periprosthetic osteolysis site. It has been further shown that there is an appearance compatible with the histology of foreign body reaction and rheumatoid arthritis around the prosthesis (11). Maloney et al demonstrated that the periprosthetic chronic inflammation damaged the prosthesis-bone relationship (12). Glant et al established findings supporting this theory (13). All these findings suggest that aseptic loosening is an intraarticular inflammatory response (14-17). As a result, it was believed that there would be an increase in the blood levels of inflammatory parameters.

The review of the literature reveals several studies on the relationship between the hematological parameters, NLR, PLR, and MLR and malignancies, chronic diseases, obesity, and systemic inflammation (18-24). Gao et al investigated the relationship between NLR, PLR, and MLR and the development of knee osteoarthritis on 119 patients, and established a statistically significant relationship when compared with control groups. The authors found MLR to have a higher diagnostic value than the other two parameters did (25). Shi et al found PLR to be associated with the development of knee osteoarthritis. Büyükavci et al demonstrated the relationship between NLR and the development of osteoarthritis (26).

The study by Trimula et al conducted on 538 patients regarding the development of periprosthetic joint infection after total knee arthroplasty concluded that TLR was a statistically significant parameter (27). In addition, Zhao et al pointed out that NLR and PLR were closely associated with early periprosthetic joint infection (28). These studies showed the relationship between hematological parameters and systemic inflammation. When we reviewed the literature, we did not find any study on the relationship between NLR, PLR, and MLR, i.e., hematological parameters, and the development of aseptic loosening. In our study, we established a significant relationship between the hematological parameter MLR and aseptic loosening. We believe that the use of this parameter in the diagnosis of aseptic loosening would be useful in determining the clinical diagnosis. Furthermore, our study is important in determining the cut-off value for this parameter.

Our study has some limitations. First, it is a retrospective study. However, the impact on the hematological data following the surgery was not examined. In addition, although the number of patients appears limited, it is believed to be sufficient to homogenize both groups.

\section{Conclusion}

Aseptic loosening is a process associated with chronic inflammation. MLR can be used to help initiating the diagnosis after total knee arthroplasty and the diagnosis can be then finalized with other auxiliary diagnostic methods.

\section{References}

1. Ethgen $\mathrm{O}$ et al. Health-related quality of life in total hip and total knee arthroplasty. A qualitative and systematic review of the literature. J Bone Joint Surg Am 2004; 86 (5): 963-974.

2. Patil $\mathbf{S}$ et al How do knee implants perform past the second decade? Nineteen- to 25-year followup of the Press-fit Condylar design TKA. Clin Orthop Relat Res 2015; 473 (1): 135-140.

3. Kremers HM et al. Comparative long-term survivorship of uncemented acetabular components in revision total hip arthroplasty. J Bone Joint Surg Am 2012; 94 (12): e82.

4. National Joint Replacement Registry, A.O.A., 2018 Hip, Knee \& Shoulder Arthroplasty Annual Report 2018.

5. Marx A et al. Comparison of subtraction arthrography, radionuclide arthrography and conventional plain radiography to assess loosening of total knee arthroplasty. Biomed Tech (Berl) 2005; 50 (5): 143-147.

6. Smith SL, Wastie ML, Forster I. Radionuclide bone scintigraphy in the detection of significant complications after total knee joint replacement. Clin Radiol 2001; 56 (3): 221-224.

7. Dennis DA. Evaluation of painful total knee arthroplasty. J Arthroplasty 2004; 19 (Suppl 1): 35-40.

8. Hirschmann MT et al. Clinical value of SPECT/CT for evaluation of patients with painful knees after total knee arthroplasty - a new dimension of diagnostics? BMC Musculoskelet Disord 2011; 12: 36.

9. Claassen L et al. Diagnostic value of bone scintigraphy for aseptic loosening after total knee arthroplasty. Technol Health Care 2014; 22 (5): 767-773.

10. Lee DH et al. Causes and Clinical Outcomes of Revision Total Knee Arthroplasty. Knee Surg Relat Res 2017; 29 (2): 104-109.

11. Maguire JK Jr, Coscia MF, Lynch MH. Foreign body reaction to polymeric debris following total hip arthroplasty. Clin Orthop Relat Res 1987; 216: 213-223.

12. Maloney WJ et al. Isolation and characterization of wear particles generated in patients who have had failure of a hip arthroplasty without cement. J Bone Joint Surg Am 1995; 77 (9): 1301-1310.

13. Glant TT, Jacobs JJ. Response of three murine macrophage populations to particulate debris: bone resorption in organ cultures. J Orthop Res 1994; 12 (5): 720-731.

14. Dapunt $U$ et al. Infectious versus non-infectious loosening of implants: activation of $\mathrm{T}$ lymphocytes differentiates between the two entities. Int Orthop 2014; 38 (6): 1291-1296.

15. Pajarinen $\mathbf{J}$ et al. Interaction of Materials and Biology in Total Joint Replacement - Successes, Challenges and Future Directions. J Mater Chem B 2014; 2 (41): 7094-7108.

16. Nich $\mathbf{C}$ et al. Macrophages-Key cells in the response to wear debris from joint replacements. J Biomed Mater Res A 2013; 101 (10): 3033-3045. 
17. Jämsen $\mathbf{E}$ et al. Characterization of macrophage polarizing cytokines in the aseptic loosening of total hip replacements. J Orthop Res 2014; 32 (9): 1241-1246.

18. Guthrie GJ et al. The systemic inflammation-based neutrophil-lymphocyte ratio: experience in patients with cancer. Crit Rev Oncol Hematol 2013; 88 (1): 218-230.

19. Imtiaz $\mathbf{F}$ et al. Neutrophil lymphocyte ratio as a measure of systemic inflammation in prevalent chronic diseases in Asian population. Int Arch Med 2012; 5 (1): 2 .

20. Furuncuoğlu $Y$ et al. How obesity affects the neutrophil/lymphocyte and platelet/lymphocyte ratio, systemic immune-inflammatory index and platelet indices: a retrospective study. Eur Rev Med Pharmacol Sci 2016; 20 (7): 1300-1306.

21. Yang $\mathbf{T}$ et al. Lymphocyte to monocyte ratio and neutrophil to lymphocyte ratio are superior inflammation-based predictors of recurrence in patients with hepatocellular carcinoma after hepatic resection. J Surg Oncol 2017; 115 (6): 718-728.

22. Tezol $\mathbf{O}$ et al. Value of neutrophil-to-lymphocyte ratio, monocyteto-lymphocyte ratio, platelet-to-lymphocyte ratio and red blood cell distribution width in distinguishing between reactive lymphadenopathy and lymphoma in children. Bratisl Med J 2020; 121 (4): 287-292.
23. Yilmaz MO, Keles FO. The relationship between neutrophil/lymphocyte ratio and clinical and radiological findings in patients with nonfunctional adrenal incidentaloma. Bratisl Med J 2021; 122 (7): 493-496.

24. Zahorec R. Neutrophil-to-lymphocyte ratio, past, present and future perspectives. Bratisl Med J 2021; 122 (7): 474-488.

25. Gao K et al. Diagnostic value of the blood monocyte-lymphocyte ratio in knee osteoarthritis. J Int Med Res 2019; 47 (9): 4413-4421.

26. Büyükavci R, Aktürk S, Sağ S. Comparison of blood platelet distribution width and neutrophil-lymphocyte ratio in patients with different grades of knee osteoarthritis. J Back Musculoskelet Rehabil 2018; 31 (6): 1035-1039.

27. Tirumala $\mathbf{V}$ et al. Diagnostic Utility of Platelet Count/Lymphocyte Count Ratio and Platelet Count/Mean Platelet Volume Ratio in Periprosthetic Joint Infection Following Total Knee Arthroplasty. J Arthroplasty 2021; 36 (1): 291-297.

28. Zhao G et al. Predictive values of the postoperative neutrophil-to-lymphocyte ratio, platelet-to-lymphocyte ratio, and lymphocyte-to-monocyte ratio for the diagnosis of early periprosthetic joint infections: a preliminary study. J Orthop Surg Res 2020; 15 (1): 571.

Received June 29, 2021. Accepted July 5, 2021. 